\title{
IncAKHE enhances cell growth and migration in hepatocellular carcinoma via activation of NOTCH2 signaling
}

Guanqun Huang ${ }^{1}$, Hui Jiang ${ }^{2}$, Ye Lin ${ }^{3}$, Yanpeng Wu' ${ }^{1}$, Weilong Cai ${ }^{1}$, Boyun Shi ${ }^{2}$ Yuanwei Luo ${ }^{1}$, Zhixiang Jian $^{3}$ and Xinke Zhou ${ }^{2}$

\begin{abstract}
Hepatocellular carcinoma is the sixth most common cancer and gives rise to numerous deaths around the world every year. However, the molecular mechanism that controls hepatocarcinogenesis remains largely unknown. Here we found out an uncharacterized long noncoding RNA named InCAKHE. We found that IncAKHE was highly expressed in hepatocellular carcinoma tissues. IncAKHE depletion remarkably impaired the abilities of cell proliferation, migration, and invasion in hepatocellular carcinoma while promgoogoting cell apoptosis. Moreover, higher expression level of IncAKHE in hepatocellular carcinoma tissues was associated with more clinical severity and lower survival rates. Mechanistically, IncAKHE cooperated with YEATS4 to enhance the activation of NOTCH2 signaling which is usually abnormally upregulated in hepatocellular carcinoma. In conclusions, our study showed that IncAKHE may promote tumor progression in HCC and serve as a novel target for HCC treatment.
\end{abstract}

\section{Introduction}

Hepatocellular carcinoma (HCC) is the sixth most common cancer and gives rise to numerous deaths around the world every year ${ }^{1,2}$. Currently, the curative therapies for HCC include radiofrequency ablation (RFA), transarterial chemoembolization (TACE) and intraarterial radiotherapy (SIRT) ${ }^{3,4}$. For advanced HCC treatment, only surgery and local ablative therapies seem effective. Although some advance has been made on the methods for HCC treatment in recent years, the 5-year overall survival rate is still under $25 \%^{5}$. Therefore, developing new curative therapies for HCC is very necessary and urgent. HCC occurrence is a multistep and complex

\footnotetext{
Correspondence: Zhixiang Jian (sdf1984365@126.com) or

Xinke Zhou (nihaeoj1465@126.com)

'Department of general surgery, the Fifth Affiliated Hospital of Guangzhou Medical University, Guangzhou 510700, China

${ }^{2}$ Department of Abdominal Oncology, the Fifth Affiliated Hospital of Guangzhou Medical University, Guangzhou 510700, China

Full list of author information is available at the end of the article

These authors contributed equally: Guanqun Huang, Hui Jiang, Ye Lin.

Edited by E. Candi
}

process associated with a variety of genetic mutations or epigenetic alterations $^{6-8}$. Many efforts have been made to explore the potential mechanisms. However, the molecular mechanisms still remain elusive. Dysregulation of gene expression is proven to be essential for hepatocarcinogenesis ${ }^{7}$. So identifying genes that promote HCC development and progression is pivotal for revealing the mechanisms of hepatocarcinogenesis and developing effective therapies.

Long noncoding RNAs (lncRNAs) are a major class of transcripts of longer than 200 nucleotides (nt) and cannot $\operatorname{code~protein~}^{9,10}$. Recent researches showed that lncRNAs have important functions and were involved in diverse biological processes, such as development, immunity, and cancer $^{11-15}$. LncRNAs perform functions in many kinds of ways, including regulating chromatin accessibility and gene expression ${ }^{16}$. For example, HOTAIR inhibits JAM2 transcription initiation by interacting with Ezh2 to reduce the chromatin accessibility ${ }^{17}$. Dysregulation of lncRNA expression may lead to many human diseases, especially

\section{(c) The Author(s) 2018}

(c) (i) Open Access This article is licensed under a Creative Commons Attribution 4.0 International License, which permits use, sharing, adaptation, distribution and reproduction cc) in any medium or format, as long as you give appropriate credit to the original author(s) and the source, provide a link to the Creative Commons license, and indicate if changes were made. The images or other third party material in this article are included in the article's Creative Commons license, unless indicated otherwise in a credit line to the material. If material is not included in the article's Creative Commons license and your intended use is not permitted by statutory regulation or exceeds the permitted use, you will need to obtain permission directly from the copyright holder. To view a copy of this license, visit http://creativecommons.org/licenses/by/4.0/. 
tumor $^{18}$. In most cancers, expression levels of many lncRNAs are abnormal. Therefore, exploring the functions of lncRNAs seems to be helpful for tumor treatment. However, the roles of large amounts of lncRNAs involved in tumorigenesis have not been defined. And the mechanisms by which lncRNAs function are still largely unknown.

In this study, we screened out a new lncRNA named as IncAKHE that was upregulated in HCC tissues compared to paired peritumor. We found that lncAKHE knockdown impaired cell proliferation and migration, and increased cell apoptosis. In mechanism, we found that lncAKHE interacted with YEATS4 to enhance NOTCH2 signaling activation which then promotes HCC progression. What's more, patients with higher lncAKHE expression had lower survival rates. In summary, our study revealed a new mechanism of hepatocarcinogenesis and lncAKHE may serve as a potential target for HCC therapy.

\section{Results \\ IncAKHE is upregulated in HCC tissues}

To explore how lncRNAs regulate HCC progression, we analyzed an online-available microarray dataset about lncRNAs expressed in HCC (GSE27462) ${ }^{19}$. We screened out upregulated or downregulated lncRNAs in all five HCC samples compared to paired peritumor tissues (Fig. 1a). Then we focused on a most upregulated and uncharacteristic lncRNA (AK056594 located on chr8: $67,245,089 \sim 67,247,203)$. We named it as lncAKHE for abbreviation of AK056594 and highly expression in HCC. Then we analyzed the expression of lncAKHE in 60 pairs of HCC and peritumor samples. We found that lncAKHE was upregulated in HCC tissues (Fig. 1b). To further confirm it, we chose five pairs of HCC samples for Northern bot and got the same result (Fig. 1c). What's more, we used biotin-labeled lncAKHE specific probes for in situ hybridation (ISH) and RNA Fluorescence in situ hybridation (RNA FISH), and found that lncAKHE was highly expressed in HCC samples (Fig. 1d, e). Besides, according to qPCR, lncAKHE was also expressed higher in HCC cell lines including Hep3B, HepG2, 7402, Huh7, MHCC97H, and HCCLM3 cells compared to human normal hepatocyte LO2 (Fig. 1f). In sum, IncAKHE was upregulated in HCC tissues.

\section{IncAKHE is positively correlated with HCC severity}

We further divided 60 pairs of HCC samples into three groups according to AJCC TNM classification. We found that lncAKHE was expressed higher in samples of stage II and Stage III (Fig. 2a). Besides, more lncAKHE was expressed in early HCC (eHCC) and advanced (aHCC) samples than normal liver tissues (Fig. 2b, c). Then we grouped these samples into high and low expression subsets according to lncAKHE expression. Kaplan-Meier survival analysis was performed. We found that HCC patients with higher lncAKHE expression possessed poorer survival rates (Fig. 2d, e). Collectively, lncAKHE is positively correlated with $\mathrm{HCC}$ severity.

\section{IncAKHE knockdown inhibits tumor cell proliferation and promotes apoptosis}

We then explored the effect of lncAKHE on tumor cells. We collected HCC samples and cultured them. By infecting tumor cells from sample \#1 and \#4 with virus containing shlncAKHE, we successfully knocked down lncAKHE as shown by qPCR and Northern blot (Fig. 3a, b). According to MTS assays, we found that IncAKHEdepleted tumor cells showed impaired proliferation ability (Fig. 3c). What's more, lncAKHE-silenced cells formed less clones (Fig. 3d) and less cells entered into $\mathrm{S}$ phase (Fig. 3e). Consistently, lncAKHE knockdown decreased the expression of proliferation-related proteins including CYCLIN D1 and PCNA (Fig. 3f). On the other hand, lncAKHE depletion also promoted cell apoptosis (Fig. 3g). In a word, lncAKHE knockdown seriously inhibited cell proliferation and promoted apoptosis.

\section{IncAKHE knockdown impairs tumor cell migration and invasion}

We found a significant correlation between lncAKHE expression and TNM stages. We subsequently conducted cell migration and invasion assays with lncAKHE-silenced tumor cells to determine the potential relationship between IncAKHE expression and metastasis. We found that lncAKHE knockdown remarkably reduced the migration and invasion potential of tumor cells (Fig. 4a, b). Consistently, lncAKHE knockdown also decreased the expression levels of SNAI1, MMP2, and MMP9 (Fig. 4c). Summarily, our study indicated that lncAKHE may promote cell metastasis in HCC patients.

\section{IncAKHE promotes tumor propagation in vivo}

To further explore the role of lncAKHE in vivo, we performed a xenograft nude mouse experiment via subcutaneously injecting shCtrl or shlncAKHE HCC sample cells. We found that lncAKHE knockdown significantly delayed the tumor growth (Fig. 5a). And the tumor weights were also decreased on 30th day post injection after IncAKHE depletion (Fig. 5b). Furthermore, the proliferation and invasion abilities of formed tumors derived from lncAKHE-silenced tumor cells were also impaired (Fig. 5c). Consistently, knockdown of lncAKHE also led to increased apoptosis in formed tumor cells, as higher levels of cleaved caspase3 were observed in shlncAKHE tumor cells than in control group (Fig. 5d). To explore how lncAKHE regulates tumor metastasis in vivo, we intravenously injected $5 \times 10^{5} \mathrm{WT}$ or shlncAKHE sample cells into lateral tail veins of recipient 
a

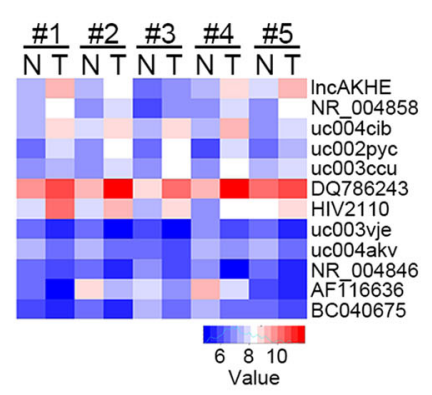

d

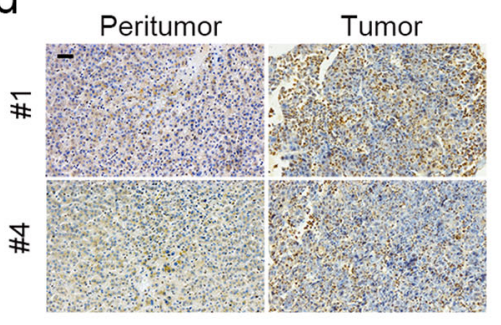

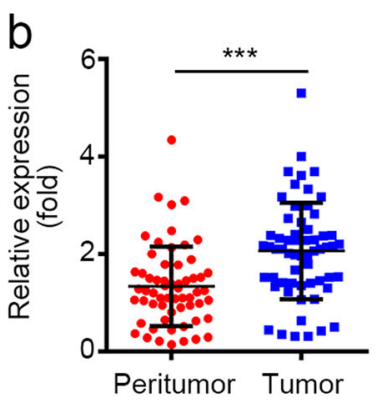

C

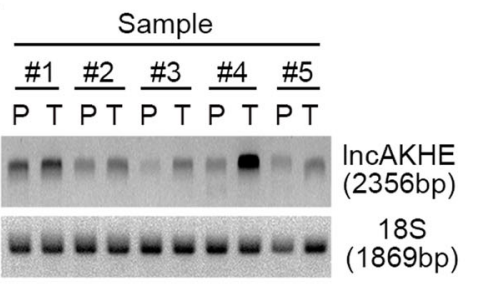

e

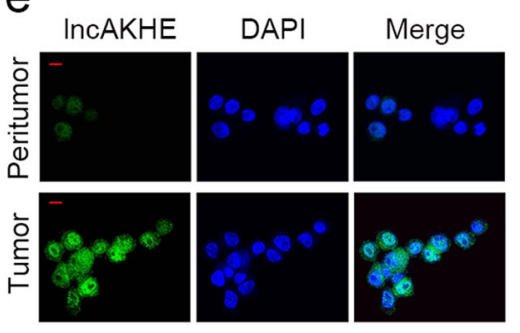

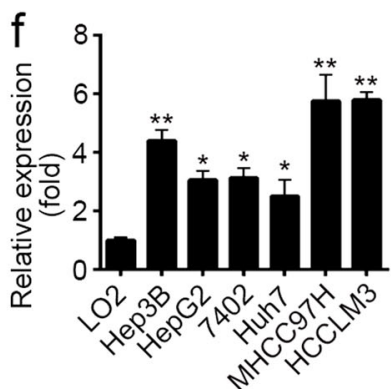

Fig. 1 LncAKHE is upregulated in HCC tissues. a Microarray data in GSE27462 were analyzed. Differentially expressed IncRNAs were listed between HCC tissues and paired peritumor samples. b 60 pairs of peritumor and tumor samples were collected and total RNAs were extracted. Then the expression levels of IncAKHE were measured by RT-qPCR. c Expression levels of IncAKHE and 18S (loading control) were analyzed in peritumor and tumor tissues. Samples from \#1 to \#5 were chosen for Northern blot. IncAKHE and $18 \mathrm{~S}$ probes were biotin-labeled. P peritumor, T tumor. d IncAKHE was expressed higher in HCC samples as shown by RNA hybridization in situ (ISH). IncAKHE probe was biotin-labeled. Scale bars $=100 \mu$ m. e The expression level of IncAKHE was checked in tumor cells by fluorescence in situ hybridization (RNA FISH). Scale bars $=10 \mu \mathrm{m}$. $\mathbf{f}$ Total RNAs were extracted from HCC cell lines. IncAKHE expression was examined by RT-qPCR. Data is from three independent experiments and expressed as mean \pm SD. ${ }^{*} p<0.05,{ }^{* *} p<0.01$, and ${ }^{* * *} p<0.001$

mice. The mice were sacrificed 4 weeks later, and the lung metastases were analyzed. As shown, lncAKHE knockdown decreased the nodules in the lung (Fig. 5e).

\section{LncAKHE interacts with YEATS4}

Previous studies showed that lncRNA can associates with proteins to regulate gene expression ${ }^{14,20}$. To screen out potential lncAKHE-interactive proteins, we performed RNA pulldown and silver staining with $\mathrm{HCC}$ sample cell lysates, followed by identification with mass spectrometry (MS) (Fig. 6a). YEATS4, a transcription activator, was identified as a potential interactive protein. We validated their interaction by RNA pulldown and RNA immune-precipitation (RNA IP) assays (Fig. 6b, c). Immunofluorescence also showed that IncAKHE colocalized with YEATS4 in HCC sample cells (Fig. 6d). Domain mapping also indicated that the region of $\mathrm{nt}$ : $1201 \sim 1600$ in lncAKHE is indispensible for its interaction with YEATS4 (Fig. 6e).

\section{LncAKHE activates NOTCH2 pathway}

Accumulating evidences indicated that NF- $\mathrm{B}, \mathrm{Wnt} / \beta-$ catenin, $\mathrm{NOTCH}$, and Hedgehog signaling pathways play essential roles in the development and progression of $\mathrm{HCC}^{21,22}$. To explore the molecular mechanism of
lncAKHE function, we analyzed the effect of lncAKHE on NF-kB, Wnt/ $\beta$-catenin, NOTCH, and Hedgehog signalings. We explored the expression of their target genes (HIF1A and VEGFA for NF-kB, MYC and TCF1 for Wnt/ $\beta$-catenin, NOTCH2, HES6 and HEY1 for NOTCH, GLI1 and GLI3 for Hedgehog) by qPCR in Hep3B, Huh7, and $\mathrm{MHCC} 97 \mathrm{H}$ cells, and found that the $\mathrm{NOTCH} 2$ signaling was downregulated after lncAKHE knockdown in these three cell lines (Fig. 7a). The same result was observed in HCC sample cells (Fig. 7b). Furthermore, we checked the protein levels of NOTCH2, YEATS4, HEY1, and HES6 in tumor tissues of the xenograft experiment. We found that the expression levels of NOTCH2, HEY1, and HES6 were lower in lncAKHE-silenced HCC cell-derived tumor tissues than in control tumor tissues, whereas YEATS4 expression was not affected (Fig. 7c). IHC staining also showed that lncAKHE depletion led to reduced expression of HES6 and HEY1 in lncAKHE-silenced tumor tissues (Fig. 7d).

Then we overexpressed lncAKHE in Hep3B and HCC sample cells. We found that lncAKHE overexpression activated NOTCH2 signaling (Fig. 7e, f). LncAKHE overexpression promoted NOTCH2, HEY1, and HES6 expression in Hep3B and HCC sample cells (Fig. 7e, f). Consistently, overexpressing lncAKHE promoted NOTCH2 

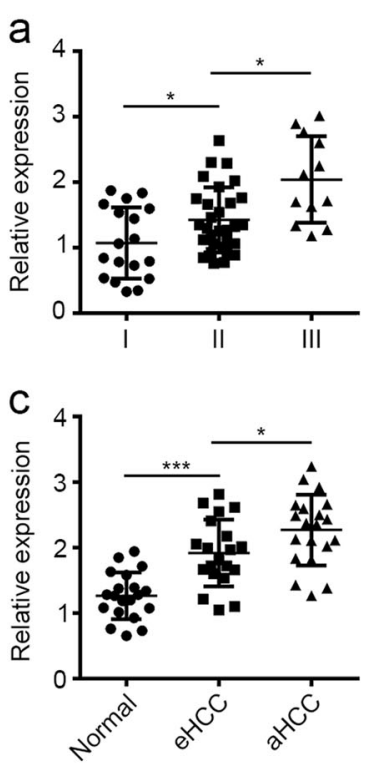

b

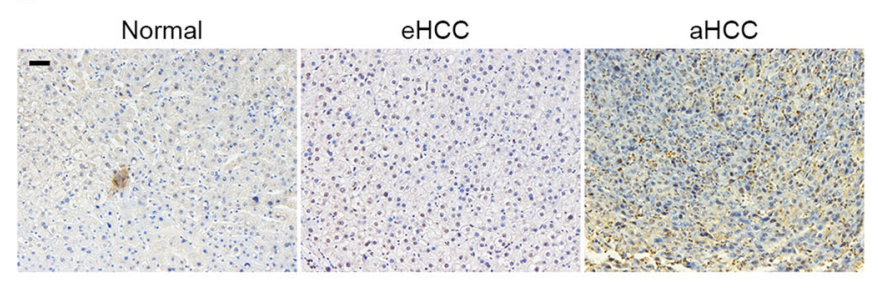

d

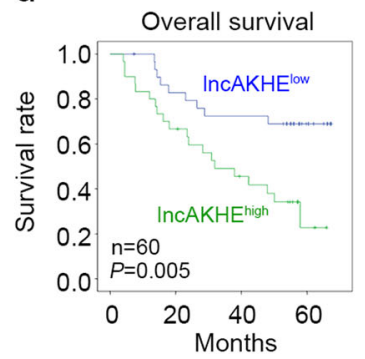

Fig. 2 LncAKHE is positively correlated with HCC severity. a HCC samples were divided into three groups based on the clinical stage (18 samples in stage I, 30 samples in Stage II, and 12 samples in stage III). Then the expression of IncAKHE was analyzed by RT-qPCR. b IncAKHE expression was checked by ISH in non-tumor, early HCC (eHCC) and advanced HCC (aHCC) tissues. Scale bar $=100 \mu \mathrm{m}$. c Total RNAs were extracted from non-tumor, eHCC, and aHCC samples. IncAKHE expression was analyzed by RT-qPCR. $\mathbf{d}$, e The survival rates in patients with higher IncAKHE expression were lower. Sixty HCC samples were divided into two groups based on the levels of IncAKHE expression. Then Kaplan-Meier survival analyses were conducted. Data is from three independent experiments and expressed as mean \pm SD. ${ }^{*} p<0.05,{ }^{* *} p<0.01$, and ${ }^{* * *} p<0.001$

expression and entry into nucleus (Fig. 7g). Then we analyzed the relationship between $\mathrm{NOTCH} 2$ signaling activation and lncAKHE expression in HCC samples and found that lncAKHE expression was positively correlated with that of HES6 or HEY1 (Fig. 7h, i).

\section{IncAKHE recruits YEATS4 to initiate NOTCH2 expression and activate NOTCH2 signaling}

To further explore how IncAKHE activates NOTCH2 signaling, we conducted chromatin isolation by RNA purification (CHIRP) assays to define whether lncAKHE directly regulates $\mathrm{NOTCH} 2$ expression. We found that lncAKHE enriched on NOTCH2 promoter (region of $-900 \sim-600 \mathrm{bp}$ from TSS) (Fig. 8a). We above demonstrated that lncAKHE associated with YEATS4 in HCC. To further explore whether YEATS4 also regulates $\mathrm{NOTCH} 2$ expression, we performed ChIP assays and found that YEATS4 bound to the same region of NOTCH2 promoter $(-900 \sim-600$ bp from TSS $)$ (Fig. 8b). Surprisingly, lncAKHE knockdown seriously impaired the enrichment of YEATS4 on NOTCH2 promoter (Fig. 8c), which indicated that lncAKHE and YEATS4 may promote NOTCH2 transcription coordinately. Consistently, overexpressing YEATS4 or lncAKHE really promoted the enrichment of RNA Pol II on $\mathrm{NOTCH} 2$ promoter and NOTCH2 mRNA level (Fig. 8d, e). Nevertheless, YEATS4 knockdown abrogated the effect of IncAKHE on NOTCH2 expression (Fig. 8d, e). Above results showed that lncAKHE recruited YEATS4 to $\mathrm{NOTCH} 2$ promoter, initiated $\mathrm{NOTCH} 2$ transcription and then activated $\mathrm{NOTCH} 2$ pathway.

To determine whether NOTCH2 pathway is dispensable for the function of IncAKHE in HCC, we inhibited NOTCH2 pathway in lncAKHE-overexpressing cells. As shown, overexpressing lncAKHE promoted the expression of proliferaton- and metastasis-related proteins while inhibition of NOTCH2 pathway with IMR-1 abrogated it (Fig. 8f). Summarily, our research showed that lncAKHE recruited YEATS to initiate NOTCH2 expression and activate $\mathrm{NOTCH} 2$ signaling pathway. Then abnormal NOTCH2 signaling activation is responsible for lncAKHE-mediated HCC cell proliferation, migration, and invasion at least partially.

\section{Discussion}

Hepatocellular carcinoma (HCC) is the sixth most common cancer and gives rise to numerous deaths around the world every year ${ }^{23,24}$. Although some advance has been made in the methods for HCC treatment recent years, the 5-year overall survival rate is still very low. Many studies have shown that some genetic mutations or abnormal activation of signaling pathways lead to hepatocarcinogenesis. For example, Pei et al. reported that FGF8 promotes HCC growth via activation of EGFR ${ }^{25}$. Activation of $W n t / \beta$-catenin leads to cell proliferation in $\mathrm{HCC}^{26}$. However, the molecular mechanism of 

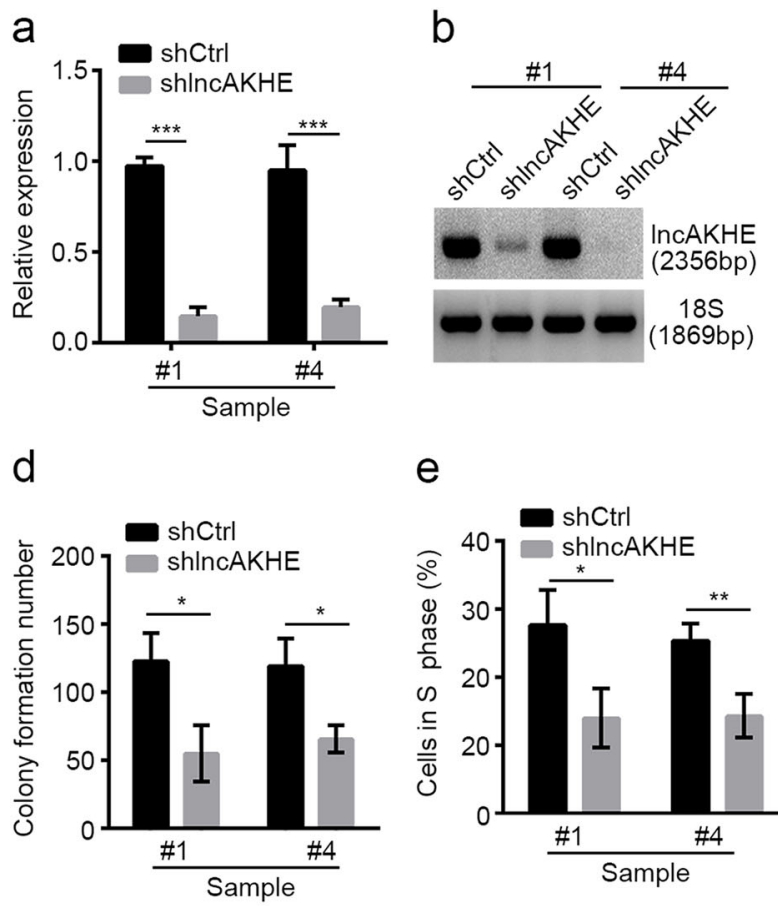

e

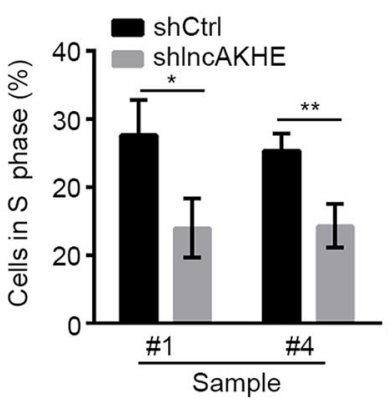

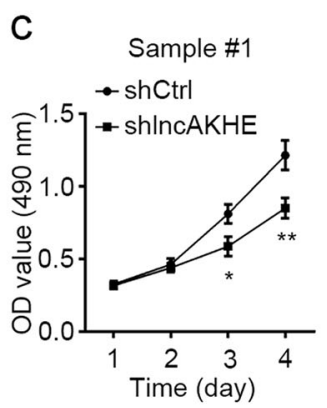

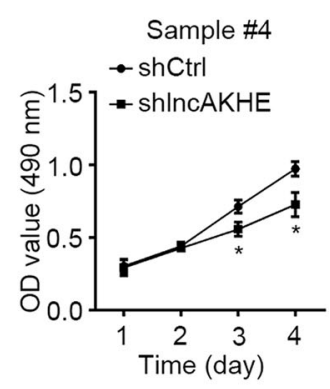

f

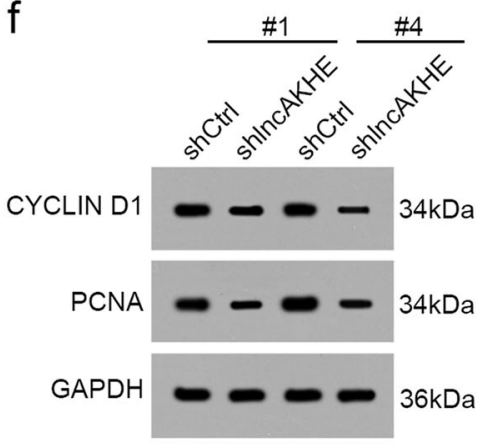

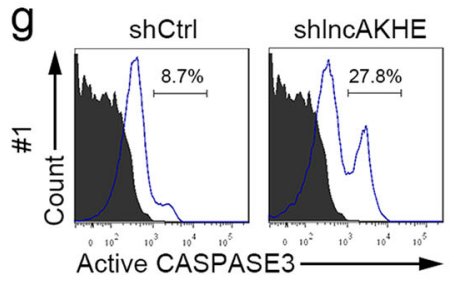

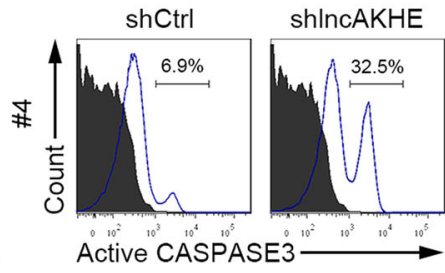

$=\lg G$

- Anti-active CASPASE3

Fig. 3 LnCAKHE knockdown inhibits tumor cell proliferation and promotes apoptosis. a, b Tumor cells from sample \#1 and \#4 were cultured and infected with pSICOR-GFP-shlncAKHE lentivirus. Then GFP+ cells were isolated by FACS. The efficiency of IncAKHE silence was analyzed by RTqPCR (a) and Northern blot (b). Cells infected with scramble shRNA were chosen for control. $\mathbf{c}$, $\mathbf{d}$ The proliferative capacity of shCtrl or shlncAKHE sample cells were measured by MTS assays (c) and colony formation assays (d). e The percentage of shCtrl or shlncAKHE sample cells in S phase was examined by FACS. $\mathbf{f}$ The protein levels of CYCLIN D1 and PCNA were decreased in shlncAKHE sample cells. GAPDH was chosen as loading control. $\mathbf{g}$ More active CASPASE3 ${ }^{+}$cells appeared after InCAKHE depletion as shown by FACS analysis. Data is from three independent experiments and expressed as mean \pm SD. ${ }^{*} p<0.05$ and ${ }^{* *} p<0.01$

hepatocarcinogenesis still remains to be explored. In our study, we screened out a new lncRNA named lncAKHE that was highly expressed in HCC tissues. LncAKHE knockdown significantly inhibited cell proliferation and invasion in HCC. Moreover, lncAKHE-silenced tumor cells showed reduced growth ability in vivo. Therefore, lncAKHE has great promise as a novel diagnostic and prognostic marker.

Accumulated evidences showed that there was a closely relationship between IncRNAs expression and tumorigenesis, metastasis or prognosis ${ }^{27-30}$. LncRNAs can serve as tumor suppressors or oncogenes by regulating gene expression or pivotal signaling pathway, such as Wnt/ $\beta$ catenin and NOTCH signaling ${ }^{31,32}$. We found that lncAKHE acted as an oncogene by activating $\mathrm{NOTCH} 2$ signaling. Through knocking down lncAKHE, we found that NOTCH signaling was downregulated. The protein levels of HEY1 and HES6, the target genes of $\mathrm{NOTCH}$ signaling, were greatly increased by lncAKHE overexpression. What is more, lncAHKE overexpression promoted NOTCH2 expression and entry into nucleus by IF staining. In the presence of $\mathrm{NOTCH}$ signaling inhibitor, lncAKHE overexpression cannot promote cell proliferation and migration in HCC. So our study showed that the function of lncAHKE in HCC was dependent on $\mathrm{NOTCH} 2$ signaling. Our study revealed a novel lncRNA in HCC and demonstrated its functions, which provides a new insight on the mechanism of HCC progression. However, we showed there were differential expression levels of lncAKHE in HCC patients, so the mechanism regulating lncAKHE expression in $\mathrm{HCC}$ remains to be further determined. Although we showed lncAKHE 

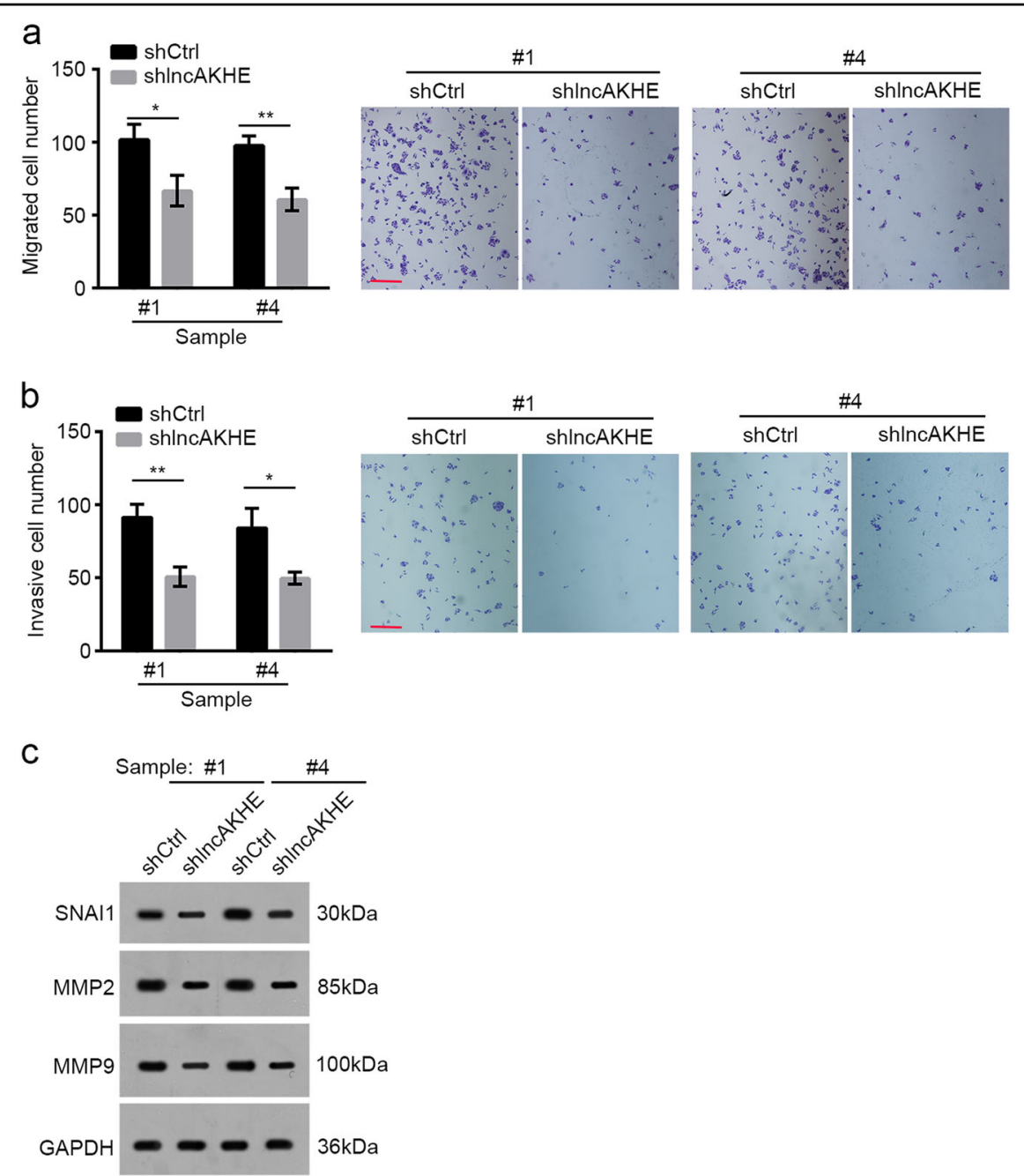

Fig. 4 LncAKHE knockdown impairs tumor cell migration and invasion. a InCAKHE knockdown decreased the migration potential of HCC sample cells in a Transwell migration assay. Scale bar $=50 \mu \mathrm{m}$. b IncAKHE knockdown significantly inhibited the invasion capacity of HCC sample cells in a Matrigel invasion assay. Scale bar $=50 \mu \mathrm{m}$. c The protein levels of SNAI1, MMP2, and MMP9 were decreased in IncAKHE-silenced tumor cells. GAPDH was chosen as loading control. Data is from three independent experiments and expressed as mean \pm SD. ${ }^{*} p<0.05$ and ${ }^{* *} p<0.01$

promoted HCC cell proliferation, migration and invasion in vitro and in vivo, whether lncAKHE promotes hepatocarcinogenesis in physical condition needs further investigation. A lncAKHE knockout mouse model may better explain the function of IncAKHE in hepatocarcinogenesis. Furthermore, NOTCH pathway has been shown to regulate the self-renewal of HCC stem cells ${ }^{21}$. Whether IncAKHE/NOTCH2 axis exerts a role on HCC stem cells also requires to be explored.

Recently, increasing evidences demonstrated that IncRNA can act as a scaffold to recruit proteins for regulation of gene expression ${ }^{9,33}$. To define the molecular mechanism of lncAKHE promoting proliferation and migration in $\mathrm{HCC}$ by $\mathrm{NOTCH} 2$ signaling, we performed RNA pulldown assays to search potential interactive proteins. We screened out YEATS4 as a candidate. Firstly, we validated the interaction of lncAKHE and YEATS4. Then we showed that lncAKHE and YEATS4 all bound to NOTCH2 promoter. Overexpressing lncAKHE or YEATS4 promoted RNA Pol II enrichment on NOTCH2 promoter, which suggested that lncAKHE and YEATS4 directly promoted $\mathrm{NOTCH} 2$ expression. Although we demonstrated lncAKHE mainly enhanced $\mathrm{NOTCH} 2$ expression in a direct manner, whether lncAKHE also upregulated $\mathrm{NOTCH} 2$ pathway activation indirectly has not been excluded. YEATS4, a transcription activator, has been reported to participate in many tumors. For example, downregulation of YEATS4 promoted cell apoptosis in colorectal cancer cells ${ }^{34}$. YEATS4 promoted cell proliferation via activating the $\mathrm{Wnt} / \beta$-catenin signaling in gastric cancer ${ }^{35}$. YEATS4 acts as an oncogene in non- 

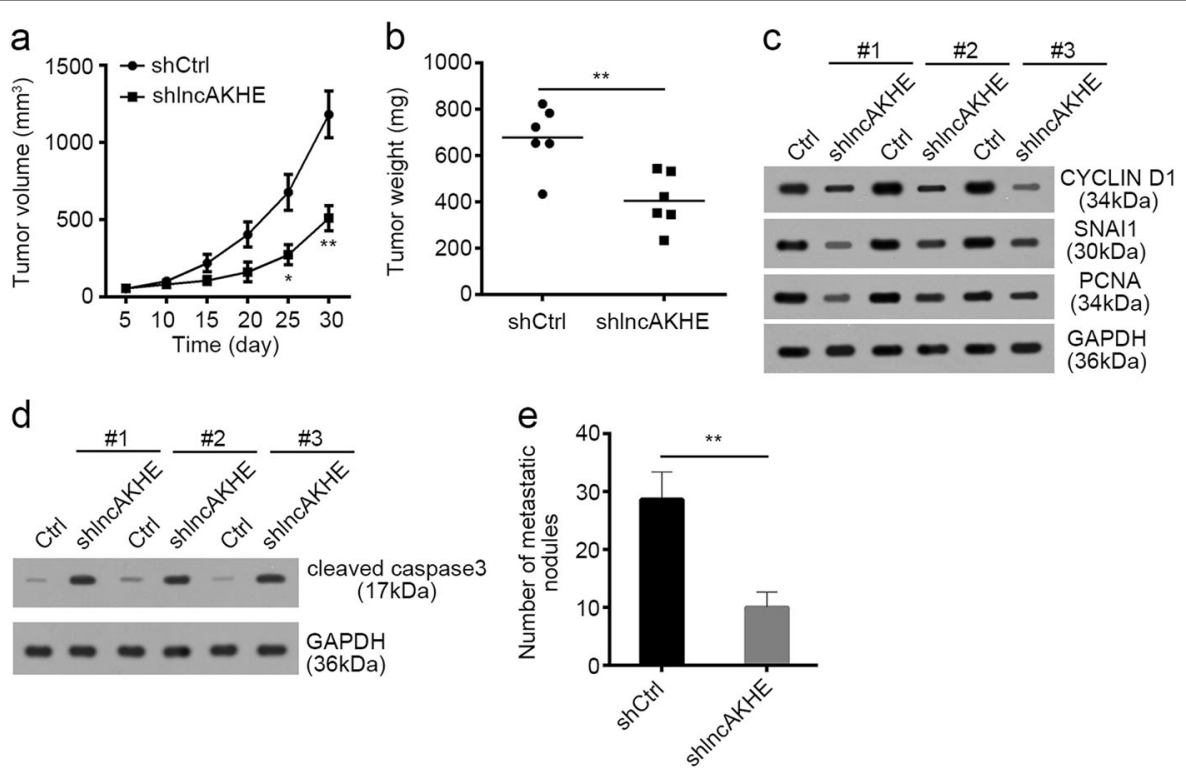

Fig. 5 LncAKHE promotes tumor propagation in vivo. a $2 \times 10^{6}$ IncAKHE-depleted or control cells were injected into nude mice. The volumes of tumors were calculated at indicative time points. LncAKHE depletion significantly impaired the rate of tumor growth in vivo. b $2 \times 10^{6}$ IncAKHEdepleted or control cells were injected into nude mice. Tumor weights were measured at 30 days post injection. The bigger tumors were originated from shCtrl tumor cells. $\mathbf{c}$ The protein levels of CYCLIN D1, SNAI1, and PCNA were checked in the formed tumors of (b). ShlncAKHE cell-derived tumors have impaired proliferation and migration abilities. $\mathbf{d}$ The protein level of cleaved caspase3 was measured in formed tumor tissues. e Quantification of the number of metastatic nodules in the lung. $n=6$ in each group. Data is from three independent experiments and expressed as mean $\pm S D .{ }^{* *} p<0.01$

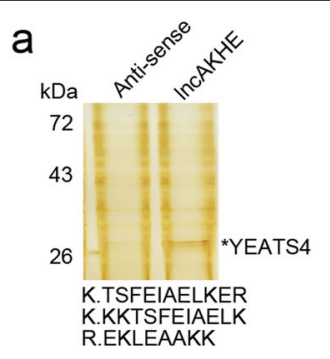

b

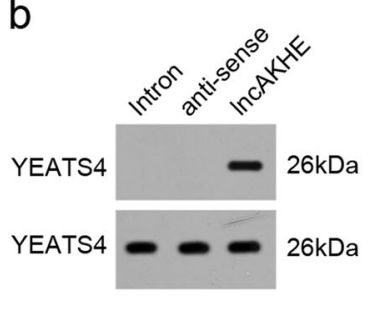

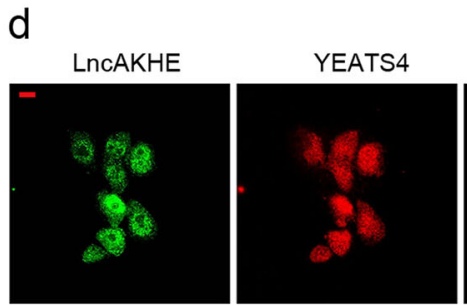
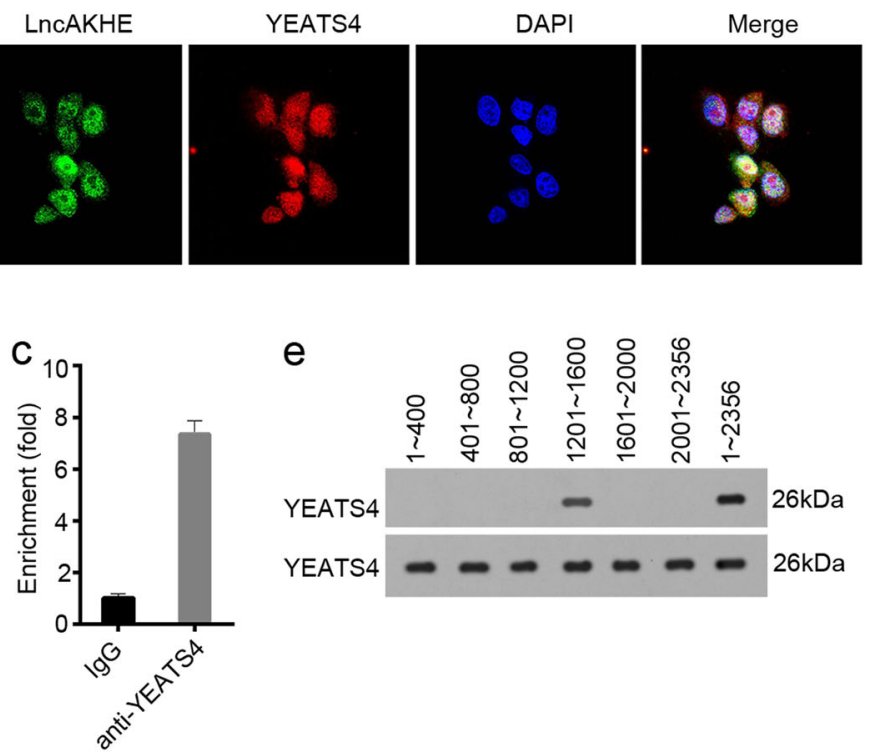

e

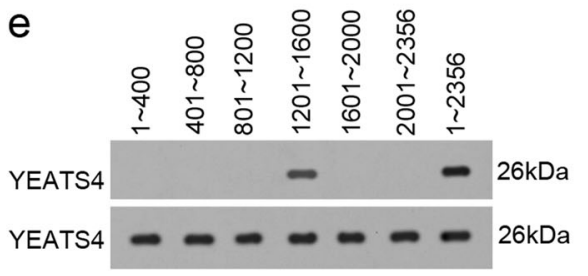

Fig. 6 LncAKHE interacts with YEATS4. a Biotin labeled InCAKHE and anti-sense controls were used for RNA pulldown assays. Then SDS-PAGE, silver staining and mass spectrometry were conducted sequentially. YEATS4 was identified as a potential interactive protein of IncAKHE. b Biotin labeled IncAKHE and control sequences were added into sample lysates and RNA pulldown assays were conducted. c Anti-YEATS4 was added into sample lysates and RNA IP assay was performed. $\mathbf{d}$ LncAKHE was co-localized with YEATS4 in the nucleus of sample cells. Green IncAKHE, Red YEATS4, Blue DAPI. Scale bar $=10 \mu \mathrm{m}$. e Domain mapping showed that region $1201 \sim 1600$ in IncAKHE was essential for its association with YEATS4. Data is from three independent experiments and expressed as mean \pm SD. ${ }^{* *} p<0.01$ 

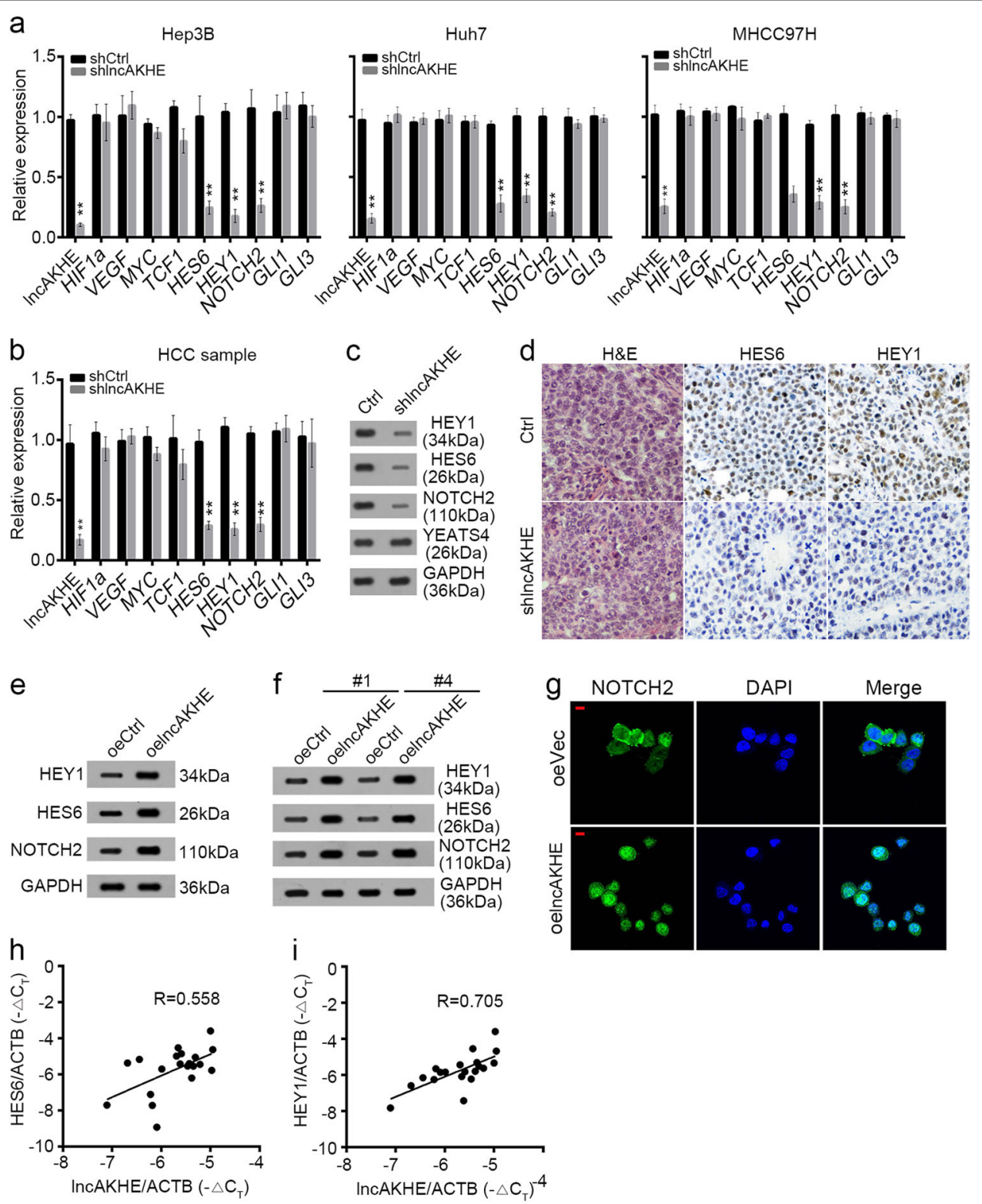

Fig. 7 LncAKHE activates NOTCH2 pathway. a LncAKHE was knocked down in Hep3B, Huh7, and MHCC97H cells. Then the target gene expression of NF-KB, Wnt/ß-catenin, NOTCH2, and Hedgehog signaling was examined by qPCR. LnCAKHE depletion inhibited NOTCH2 signaling. b NOTCH2 signaling was also impaired in IncAKHE-silenced HCC cells.c Protein levels of YEATS4, NOTCH2, HES6, and HEY1 in formed tumor tissues of the xenograft experiment. $\mathbf{d}$ Expression levels of HES6 and HEY1 in formed tumor tissues of the xenograft experiment were measured by IHC staining. e and $\mathbf{f}$ The protein levels of NOTCH2, HEY1, and HES6 were increased in IncAKHE overexpressing Hep3B (e) and HCC sample (f) cells. GAPDH was chosen as loading control. $\mathbf{g}$ LnCAKHE overexpression promotes NOTCH2 entry into nucleus in HCC sample cells. Scale bar $=10 \mu \mathrm{m}$. $\mathbf{h}$ and $\mathbf{i}$ The expression levels of HES6 (h) or HEY1 (i) were positively correlated with that of InCAKHE in HCC samples. Data is from three independent experiments and expressed as mean \pm SD. ${ }^{* *} p<0.01$

small cell lung cancer ${ }^{36}$. However, the function of YEATS4 in HCC has not been defined. By analysis of YEATS4 expression, we found that YEATS4 was also highly expressed in HCC. According to our all results, YEATS4 also served as an oncogene in HCC. However, as a chromatin remodeling protein, YEATS4 will regulate a large range of gene expression via various mechanisms. Therefore, the physiological functions of YEATS4 in HCC may be not completely the same as lncAKHE, which need more investigation. And the mechanism that YEATS4 exerts functions in lncAKHEindependent manner in HCC remains to be defined. 

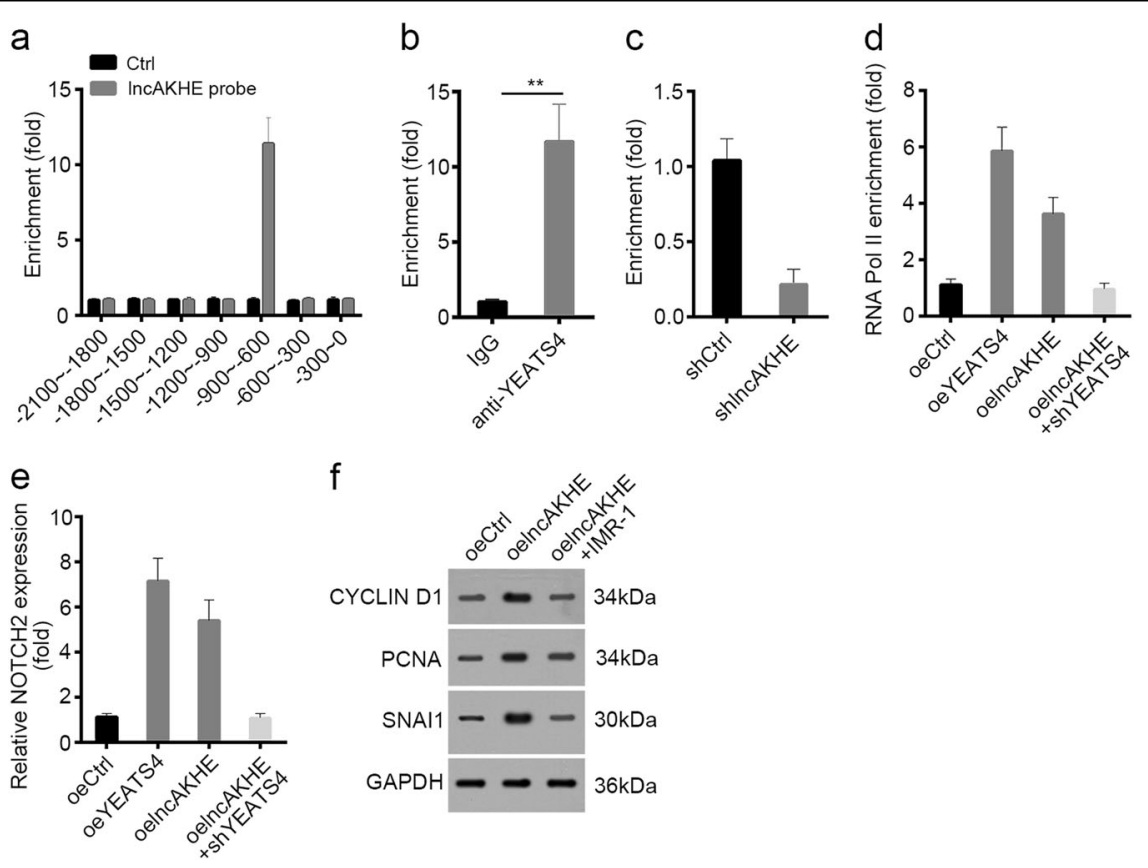

Fig. 8 LncAKHE recruits YEATS4 to initiate NOTCH2 expression and activate NOTCH2 signaling. a LncAKHE enriched on NOTCH2 promoter by CHIRP assay. LncAKHE probes were biotin-labeled. Genomes from HCC sample lysates were sonicated into fragments of $\sim 500$ bp and incubated with IncAKHE probes. $\mathbf{b}$ YEATS4 was enriched on NOTCH2 promoter as shown by ChIP assays. $\mathbf{c}$ LncAKHE knockdown impaired the enrichment of YEATS4 on NOTCH2 promoter. $\mathbf{d}$ Overexpressing YEATS4 or InCAKHE promoted the enrichment of RNA Pol II on NOTCH2 promoter. e Overexpressing YEATS4 or InCAKHE promoted the expression of NOTCH2. $\mathbf{f} L$ LnCAKHE overexpression promoted cell proliferation and migration while inhibition of NOTCH signaling by addition of IMR-1 reversed it. Data is from three independent experiments and expressed as mean \pm SD. ${ }^{* *} p<0.01$

\section{Materials and methods \\ Patient samples}

For this study, we collected 60 HCC samples from the Fifth Affiliated Hospital of Guangzhou Medical University. We listed the samples' clinical characteristics in Supplementary Table 1. For tumor cell culture, we cut $\mathrm{HCC}$ tissues into pieces and digested using collagenase IV for $50 \mathrm{~min}$ at $37^{\circ} \mathrm{C}$. We obtained consents that approve usage of these $\mathrm{HCC}$ tissues in this study from all patients. All the experiments were approved by the Fifth Affiliated Hospital of Guangzhou Medical University. The study protocol was approved by the Fifth Affiliated Hospital of Guangzhou Medical University.

\section{Cell lines}

The normal liver cell line LO2 and The HCC cell lines (Hep3B, 7402, Huh7, and HepG2) were obtained from ATCC. All cells were cultured in RPMI 1640 medium (Invitrogen, Shanghai, China) supplemented with $10 \%$ fetal bovine serum (FBS; Invitrogen) and $100 \mathrm{U} / \mathrm{ml}$ penicillin plus $100 \mu \mathrm{g} / \mathrm{ml}$ streptomycin.

\section{ShRNA-mediated interference}

shlncAKHE and shYEATS4 were designed and obtained from Invitrogen. The shRNA sequences were as follows: lncAKHE:
5'-TCCTGATTCTCTCCCTCCTCC-3',
YEATS4: 5'-AACAGTATATGTGAAACCATAT-3'. shRNAs were constructed into pSICOR-GFP plasmid. To produce lentivirus, pSICOR-GFP-shlncAKHE was transfected into 293T cells along with VSVG, RRE, and REV plasmids through a Lipofectamine 3000 kit (Invitrogen). $24 \mathrm{~h}$ later, the medium was collected and added with PEG5000. Then we collected virus by centrifugation, followed by infection into tumor cells. $\mathrm{GFP}^{+}$cells were purified by FACS and the depletion of lncAKHE or YEATS4 was validated by qPCR or WB. Control tumor cells were infected with virus containing pSICOR-GFPscramble.

\section{Antibodies}

Anti-GAPDH (5174), anti-CYCLIN D1 (2978), antiSNAI1 (3879), anti-MMP2 (87809), anti-MMP9 (13667), anti-NOTCH2 (5732), and anti-PCNA (13110) were from Cell Signaling Technology. Anti-YEATS4 (sc-81278), anti-HEY1 (sc-134362) and anti-HES6 (sc-133196) were from Santa Cruz. Anti-active CASPASE (3550914) was from BD Bioscience.

\section{Xenograft assays}

Ten male BALB/c nude mice (6 weeks old) were purchased from HFK Biosciences. Each mouse was subcutaneously injected with $2 \times 10^{6}$ shCtrl tumor cells on 
the left flank region and shlncAKHE tumor cells on the right flank region. The tumor volumes were measured at indicative time points. After 30 days, the weights of tumors were analyzed. Animal experiments were performed in accordance with relevant guidelines and regulations of the Institutional Animal Care and Use Committees at the Fifth Affiliated Hospital of Guangzhou Medical University, and protocols were approved by the Institutional Animal Care and Use Committees at the Fifth Affiliated Hospital of Guangzhou Medical University.

\section{Cell proliferation assay}

An amount of $1 \times 10^{3}$ cells were seeded in 96-well plates and cultured. Cell proliferation was measured by an MTS cell proliferation assay every other day. A volume of $20 \mu \mathrm{L}$ MTS was added and incubated for $2 \mathrm{~h}$ at $37^{\circ} \mathrm{C}$. Then the absorbance was analyzed at $490 \mathrm{~nm}$.

\section{Migration and invasion assays}

Cell migration and invasion assays have been described before $^{37}$. In brief, $2 \times 10^{3}$ cells/ well were seeded into the upper chamber of 24-well chambers and cultured in serum-free medium. The lower chamber was added medium with $10 \%$ FBS. $48 \mathrm{~h}$ later, migrating cells were fixed and stained with $0.5 \%$ crystal violet. Then three randomly fields were counted with a microscope. For invasion assay, the seeded cells were pre-coated with 500 $\mathrm{ng} / \mathrm{ml}$ Matrigel (BD). Other steps were the same as migration assay.

\section{Real-time quantitative PCR}

Total RNAs were isolated using TRIzol reagent from HCC samples according to the manufacturer's protocol. cNDA were synthesized and used for analysis of mRNA transcripts using ABI 7300 qPCR system. Relative expressions were calculated and normalized to endogenous $A C T B$. Specific primer sequences were listed in Supplementary Table 2.

\section{Chromatin immunoprecipitation (ChIP) assay}

ChIP assay were performed as described before ${ }^{38}$. In brief, HCC cells were fixed with $1 \%$ formaldehyde at $37^{\circ} \mathrm{C}$ for $10 \mathrm{~min}$. Then cells were lysed and genomes were sonicated into $\sim 500 \mathrm{bp}$ fragments. Specific antibodies were added into lysates and incubated overnight at $4{ }^{\circ} \mathrm{C}$. Then DNA fragments were enriched by Protein A Agarose/Salmon Sperm DNA (50\% Slurry) beads and purified, followed by qPCR analysis. Specific primer sequences were listed in Supplementary Table 3.

\section{LncRNA in situ hybridization}

The expression of lncAKHE in HCC tissues were measured with biotin-labeled lncAKHE probes.
Paraffinized sections were deparaffinized with xylene and $100 \%$ ethanol. Then sections were incubated with biotinlabeled probes for $18 \mathrm{~h}$ at $40^{\circ} \mathrm{C}$. DAB substrate was used for colorimetric detection of IncAKHE. Finally, the sections were co-stained with hematoxylin, followed by dehydration in graded alcohols and xylene. Biotinconjugated probes were purchased from Invitrogen. lncAKHE probe sequences as follows: \#1: $5^{\prime}$ TTGGGTTAGGCAAACACTGT-3'; \#2: 5'-ACAACAGATTGATAGTCCAT-3'; \#3: 5' -ACAATAGCACCCCAATAAGA- 3 '.

\section{Northern blot}

RNAs were isolated from HCC samples and cell lines using TRIZOL (Invitrogen). lncAKHE and 18S probes for Northern blot were achieved by Biotin RNA labeling mix (Roche). The RNA samples were separated by electrophoresis and transferred to NC membrane. Then the membranes were incubated with hydration buffer containing probes. Finally, RNA signal was detected with Chemiluminescent Nucleic Acid Detection Module (Thermo Scientific).

\section{Pulldown and mass spectrometry}

HCC lysates were incubated with biotin-labeled lncAKHE at $4{ }^{\circ} \mathrm{C}$ overnight. Then beads were added for incubation for another $4 \mathrm{~h}$. Finally, beads were collected and washed. Then SDS-PAGE was performed, followed by silver staining. Differential bands that appeared in lncAKHE lane were cut off for mass spectrometry (LTQ Orbitrap XL). Biotin-labeled lncAKHE was achieved by a T7 transcription in vitro kit (Roche).

\section{Statistical analysis}

All statistical analyses were performed using the Statistical Package for the Social Sciences version 20.0 software (SPSS Inc., Chicago, IL, USA). Survival curves were calculated using the Kaplan-Meier method and were analyzed using the log-rank test. For comparisons, oneway analyses of variance, Fisher's exact tests, chi-squared tests, and two-tailed Student's $t$-tests were performed, as appropriate. $P<0.05$ was considered statistically significant.

\section{Acknowledgements}

This work was supported by science and technology projects of Guangdong province (2017A020211031, 2016ZC0145), science and technology projects of Guangzhou Medical University (201624), the National Natural Science Foundation of China (81673206) and Medical Scientific Research Foundation of Guangdong province (A2015243).

\footnotetext{
Author details

'Department of general surgery, the Fifth Affiliated Hospital of Guangzhou Medical University, Guangzhou 510700, China. ${ }^{2}$ Department of Abdominal Oncology, the Fifth Affiliated Hospital of Guangzhou Medical University, Guangzhou 510700, China. ${ }^{3}$ Department of General Surgery, Guangdong
} 
General Hospital, Guangdong Academy of Medical Sciences, Guangzhou 510080, China

\section{Author contributions}

Guanqun Huang designed and performed experiments; Hui Jiang and Ye Lin performed experiments; Yanpeng Wu, Weilong Cai, Boyun Shi, and Yuanwei Luo analyzed the data; Zhixiang Jian and Xinke Zhou initiated the study, organized, designed, and wrote the paper.

\section{Conflict of interest}

The authors declare that they have no conflict of interest.

\section{Publisher's note}

Springer Nature remains neutral with regard to jurisdictional claims in published maps and institutional affiliations.

Supplementary Information accompanies this paper at (https://doi.org/ 10.1038/s41419-018-0554-5).

Received: 12 December 2017 Revised: 2 March 2018 Accepted: 9 March 2018

Published online: 30 April 2018

\section{References}

1. Jemal, A. et al. Global cancer statistics. CA Cancer J. Clin. 61, 69-90 (2011).

2. El-Serag, H. B. Epidemiology of viral hepatitis and hepatocellular carcinoma. Gastroenterology 142, 1264-1273 e1261 (2012).

3. Ulahannan, S. V. et al. Earlier presentation and application of curative treatments in hepatocellular carcinoma. Hepatology 60, 1637-1644 (2014).

4. Chen, C. L. et al. Bromodomain-containing protein 7 (BRD7) as a potential tumor suppressor in hepatocellular carcinoma. Oncotarget 7, 16248-16261 (2016).

5. El-Serag, H. B. Hepatocellular carcinoma. N. Engl. J. Med 365, 1118-1127 (2011).

6. Farazi, P. A. \& DePinho, R. A. Hepatocellular carcinoma pathogenesis: from genes to environment. Nat. Rev. Cancer 6, 674-687 (2006).

7. Villanueva, A., Newell, P., Chiang, D. Y., Friedman, S. L. \& Llovet, J. M. Genomics and signaling pathways in hepatocellular carcinoma. Semin. Liver Dis. 27, 55-76 (2007).

8. Glassman, M. L., de Groot, N. \& Hochberg, A. Relaxation of imprinting in carcinogenesis. Cancer Genet. Cytogenet. 89, 69-73 (1996).

9. Liu, B. et al. Long noncoding RNA IncKdm2b is required for ILC3 maintenance by initiation of Zfp292 expression. Nat. Immunol. 18, 499-508 (2017).

10. Bhan, A., Soleimani, M. \& Mandal, S. S. Long Noncoding RNA and Cancer: A New Paradigm. Cancer Res. 77, 3965-3981 (2017).

11. Li, S. et al. Zbtb7b engages the long noncoding RNA Blnc1 to drive brown and beige fat development and thermogenesis. Proc. Natl Acad. Sci. USA 114, E7111-E7120 (2017)

12. Mach, J. The Long-Noncoding RNA ELENA1 Functions in Plant Immunity. Plant Cell 29, 916 (2017).

13. Zhu, P. et al. LncBRM initiates YAP1 signalling activation to drive self-renewal of liver cancer stem cells. Nat. Commun. 7, 13608 (2016).

14. Atianand, M. K. et al. A Long Noncoding RNA lincRNA-EPS Acts as a Transcriptional Brake to Restrain Inflammation. Cell 165, 1672-1685 (2016).
15. Zhu, P. et al. Inc-beta-Catm elicits EZH2-dependent beta-catenin stabilization and sustains liver CSC self-renewal. Nat. Struct. Mol. Biol. 23, 631-639 (2016).

16. Bhan, A. \& Mandal, S. S. LncRNA HOTAIR: A master regulator of chromatin dynamics and cancer. Biochim. Biophys. Acta 1856, 151-164 (2015).

17. Gupta, R. A. et al. Long non-coding RNA HOTAIR reprograms chromatin state to promote cancer metastasis. Nature 464, 1071-1076 (2010).

18. Peng, W. X., Koirala, P. \& Mo, Y. Y. LncRNA-mediated regulation of cell signaling in cancer. Oncogene 36, 5661-5667 (2017).

19. Yang, F. et al. Long noncoding RNA high expression in hepatocellular carcinoma facilitates tumor growth through enhancer of zeste homolog 2 in humans. Hepatology 54, 1679-1689 (2011).

20. Quinn, J. J. \& Chang, H. Y. Unique features of long non-coding RNA biogenesis and function. Nat. Rev. Genet. 17, 47-62 (2016).

21. Zhu, P. et al. C8orf4 negatively regulates self-renewal of liver cancer stem cells via suppression of NOTCH2 signalling. Nat. Commun. 6, 7122 (2015).

22. $\mathrm{Xu}, \mathrm{Y}$. C. et al. LncSHRG promotes hepatocellular carcinoma progression by activating HES6. Oncotarget 8, 70630-70641 (2017).

23. Allemani, C. et al. Global surveillance of cancer survival 1995-2009: analysis of individual data for $25,676,887$ patients from 279 population-based registries in 67 countries (CONCORD-2). Lancet 385, 977-1010 (2015).

24. Altekruse, S. F., McGlynn, K. A., Dickie, L. A. \& Kleiner, D. E. Hepatocellular carcinoma confirmation, treatment, and survival in surveillance, epidemiology, and end results registries, 1992-2008. Hepatology 55, 476-482 (2012).

25. Pei, Y. et al. FGF8 promotes cell proliferation and resistance to EGFR inhibitors via upregulation of EGFR in human hepatocellular carcinoma cells. Oncol. Rep. 38, 2205-2210 (2017)

26. Chang, P. H., Sekine, K., Chao, H. M., Hsu, S. H. \& Chern, E. Chitosan promotes cancer progression and stem cell properties in association with Wnt signaling in colon and hepatocellular carcinoma cells. Sci. Rep. 8, 45751 (2017).

27. Qiu, M. T., Hu, J. W., Yin, R. \& Xu, L. Long noncoding RNA: an emerging paradigm of cancer research. Tumour Biol. 34, 613-620 (2013).

28. He, $Y$. et al. Long noncoding RNAs: novel insights into hepatocelluar carcinoma. Cancer Lett. 344, 20-27 (2014).

29. Fang, X. Y., Pan, H. F., Leng, R. X. \& Ye, D. Q. Long noncoding RNAs: novel insights into gastric cancer. Cancer Lett. 356, 357-366 (2015). (2 Pt B).

30. Mang, Y. et al. Long noncoding RNA NEAT1 promotes cell proliferation and invasion by regulating hnRNP A2 expression in hepatocellular carcinoma cells. Onco Targets Ther. 10, 1003-1016 (2017).

31. Chen, J. et al. Role of LncRNA TUG1 in intervertebral disc degeneration and nucleus pulposus cells via regulating Wnt/beta-catenin signaling pathway. Biochem. Biophys. Res. Commun. 491, 668-674 (2017).

32. Katsushima, K. et al. Targeting the Notch-regulated non-coding RNA TUG1 for glioma treatment. Nat. Commun. 7, 13616 (2016).

33. Fernando, T. R. et al. The IncRNA CASC15 regulates SOX4 expression in RUNX1-rearranged acute leukemia. Mol. Cancer 16, 126 (2017).

34. $\mathrm{Fu}, \mathrm{Q}$. et al. Downregulation of YEATS4 by miR-218 sensitizes colorectal cancer cells to L-OHP-induced cell apoptosis by inhibiting cytoprotective autophagy. Oncol. Rep. 36, 3682-3690 (2016).

35. Ji, S., Zhang, Y. \& Yang, B. YEATS domain containing 4 promotes gastric cance cell proliferation and mediates tumor progression via activating the wnt/betacatenin signaling pathway. Oncol. Res. 25, 1633-1641 (2017).

36. Pikor, L. A. et al. YEATS4 is a novel oncogene amplified in non-small cell lung cancer that regulates the p53 pathway. Cancer Res 73, 7301-7312 (2013).

37. Zhu, L. et al. LINC00052 upregulates EPB41L3 to inhibit migration and invasion of hepatocellular carcinoma by binding miR-452-5p. Oncotarget $\mathbf{8}$ 63724-63737 (2017)

38. Liu, B. et al. IL-7Ralpha glutamylation and activation of transcription factor Sall3 promote group 3 ILC development. Nat. Commun. 8, 231 (2017). 